\section{Cahiers de Narratologie}

Analyse et théorie narratives

$20 \mid 2011$

Voix off et narration cinématographique

\title{
Voix-off et commentaire dans le cinéma documentaire et ethnographique
}

\section{Silvia Paggi}

\section{OpenEdition}

1 Journals

\section{Édition électronique}

URL : https://journals.openedition.org/narratologie/6321

DOI : 10.4000/narratologie.6321

ISSN : 1765-307X

Éditeur

LIRCES

Référence électronique

Silvia Paggi, "Voix-off et commentaire dans le cinéma documentaire et ethnographique », Cahiers de Narratologie [En ligne], 20 | 2011, mis en ligne le 18 juillet 2011, consulté le 21 septembre 2021. URL: http://journals.openedition.org/narratologie/6321 ; DOI : https://doi.org/10.4000/narratologie.6321

Ce document a été généré automatiquement le 21 septembre 2021.

Article L.111-1 du Code de la propriété intellectuelle. 


\section{Voix-off et commentaire dans le cinéma documentaire et ethnographique}

\section{Silvia Paggi}

\section{Introduction}

1 Ces propos introductifs à la deuxième journée d'étude "Voix-off et narration cinématographique » proposent quelques lignes d'analyse de la voix-off dans le cinéma documentaire, notamment ethnographique. Dans ce vaste domaine, quelques extraits de films seront cités comme exemples de mise en scène de la voix-off qui, on le sait, assure avant tout dans le documentaire une fonction de commentaire. Après un bref parcours à travers les premières années de la cinématographie documentaire, du temps du muet à l'époque dite "classique ", nous montrerons la rupture de style qui intervient avec le cinéma direct pour souligner cette nouvelle approche de la voix-off, voire sa disparition.

Pour terminer, nous analyserons les principaux traits du commentaire en voix-off d'un film ethnographique, pris comme un exemple de la recherche d'équilibre et de complémentarité entre la communication artistique du cinéma, et celle, scientifique, de l'anthropologie.

3 Nous ne pouvons dans ce texte illustrer les extraits cinématographiques choisis, qui ne seront donc que cités ou brièvement décrits. Nous nous excusons auprès du lecteur de cet appauvrissement, convaincus comme nous le sommes que les mots de l'écrit ne peuvent remplacer la richesse de la communication audio-visuelle. 


\section{La relation textes-images}

4 De même que pour tous les autres sons (linguistiques et non), l'analyse de la voix-off participe de la relation textes-images qui détermine la signification de chaque élément qui compose la narration filmique.

5 Bien que l'on puisse considérer le texte en voix-off par lui-même, sa signification cinématographique repose sur la relation qu'il entretient avec ce qui est montré par les images; il s'agit du montage d'une co-occurrence ${ }^{1}$. Ainsi, notamment pour le cinéma documentaire dit «du réel», peut-on désigner cette relation comme complémentaire, redondante, ou encore concurrentielle, dans le sens où le réel est appréhendé tant par le «montré » des images que par « l'évoqué » du discours porté par le commentaire en voixoff.

6 Dans l'analyse d'une œuvre cinématographique, il est nécessaire de distinguer les éléments qui la composent selon leur statut diégétique ${ }^{2}$ ou extradiégétique. Cette distinction est particulièrement nécessaire face aux enjeux de la cinématographie documentaire - et davantage encore pour le film ethnographique dont l'approche se situe au sein des sciences sociales.

7 Le commentaire en voix-off est, presque par définition, extradiégétique, de même que la musique-off; mais bien d'autres éléments extradiégétiques peuvent faire fonction de commentaire au niveau visuel (panneaux écrits, schémas, dessins, etc.).

8 Comme tout son est audible même si la source n'est pas représentée à l'image, il n'est peut-être pas inutile de préciser que ce qui est hors-champ est diégétique, même si, dans certains cas, le spectateur peut légitimement en douter ${ }^{3}$. Il n'est peut-être pas non plus inutile de rappeler, car c'est parfois entendu autrement, que l'extradiégétique entre en relation de signification avec le diégétique : il participe donc à part entière au récit filmique. C'est notamment le cas de la voix-off, mais aussi de la musique-off, qui influence la perception notamment au niveau émotionnel. De plus, certaines informations données par des éléments extradiégétiques, la voix-off entre autres, sont supposées réelles, voire connues par des acteurs de l'univers diégétique.

Enfin, dans l'analyse de la voix-off, on ne peut pas négliger la qualité de l'oralité qu'elle porte et qui la dénote. La connotation de la voix (sexe, âge, etc.), le style de la diction, ainsi que les intonations employées contribuent largement, on le sait, à la signification du contenu du texte communiqué.

\section{Le commentaire des films documentaires muets}

10 Le premier film documentaire reconnu en tant que tel dans l'histoire du cinéma, Nanook of the North de Robert Flaherty, est de 1922, il appartient donc à l'époque du cinéma dit «muet», sans «sonore », c'est-à-dire exclusivement «visuel». Toutefois, nous savons que le cinéma muet non seulement bénéficiait souvent d'un accompagnement sonore (musical et/ou parlé) exécuté en direct pendant la projection, mais utilisait aussi l'écriture pour suppléer à son « mutisme».

11 Le documentaire Nanouk l'Esquimau, pour rappeler son titre en français, utilise donc, ainsi que le faisait le cinéma de fiction, des panneaux écrits («cartons ») juxtaposés entre les séquences d'images pour compléter le fil de la narration. Ne s'agissant que très rarement 
de l'écriture des paroles des protagonistes, ces textes écrits relevaient donc essentiellement d'une forme de commentaire.

À la grande différence du commentaire oral en voix-off, qui peut demeurer tout le long du film, les panneaux écrits ne peuvent que se juxtaposer aux séquences filmées, ce qui implique l'alternance des respectifs registres perceptifs et de signification. Dans bien des cas, le commentaire écrit des films documentaires pouvait se concentrer au début et à la fin du film, sorte de prologue et d'épilogue à la narration par images.

13 Dans Nanook of the North, Flaherty utilise l'alternance des séquences filmées avec des panneaux écrits, d'une longueur variable et sur fond d'images fixes, qui apparaissent donc de façon intermittente tout au long du film, marquant notamment le passage temporel et thématique d'une séquence à l'autre.

On remarquera en passant que l'autre éminent ancêtre du film documentaire, L'Homme à la caméra de Dziga Vertov, n'a aucune sorte de commentaire, ce qui peut sembler étonnant car son style cinématographique est sans doute moins réaliste que celui de Flaherty. L'explication est à rechercher dans l'autonomie de signification du montage d'images revendiquée dans le cadre du cinéma soviétique, autonomie à laquelle Vertov rend encore une fois hommage en réalisant ce film muet en 1929, à une époque donc où le passage au « sonore » s'était déjà accompli.

\section{La voix-off des films documentaires de l'époque « classique »}

15 Avec l'apparition du sonore ${ }^{4}$ le cinéma documentaire développe le commentaire en voixoff. On peut dire, citant Jane Gueronnet ${ }^{5}$, que le commentaire en voix-off est l'héritier du commentaire oral de l'époque du muet. La voix-off est ainsi enregistrée sur la bande son du film et non plus improvisée en direct lors de la projection.

Bien qu'il puisse assumer différentes formes, le commentaire en voix-off a une vocation narrative principalement didactique et devient souvent redondant par rapport aux images dont il double la description des actions montrées.

17 Si ce style de réalisation peut être identifié au cinéma documentaire «classique ", des années Trente jusqu'à l'après-guerre, on peut aisément constater que le commentaire en voix-off demeure et même prédomine dans la cinématographie documentaire jusqu'à présent.

On peut citer quelques films qui exemplifient des approches textuelles en voix-off de cette époque classique du documentaire.

Dans Night Mail (1936), documentaire britannique de Harry Watt et Basil Wright, la voixoff délivre un commentaire essentiellement informatif, parfois redondant avec les images. Produit par l'unité cinématographique du General Post Office (dirigée par John Grierson), ce film exalte par son commentaire - dit par Grierson lui-même - l'efficacité et la vitesse de la distribution du courrier par le train sur la ligne Londres-Glasgow : "Ces lettres ont été postées à Bletchely il y a une demi-heure ».

Des bruits de pas et autres sons supposés diégétiques - mais sans doute rajoutés en postproduction- demeurent sur un fond sonore dans lequel la voix-off est prépondérante. Cependant, la qualité du montage de ces sons « diégétiques » est plutôt inégale et finalement peu réaliste, ce qui autorise le spectateur à le ressentir comme un 
film muet sonorisé. Il est aussi étonnant d'entendre par moments des voix autres que celle off du commentaire, comme s'il s'agissait des voix des personnes filmées, dont en revanche on peut remarquer à l'image l'absence d'articulation labiale du son.

La redondance appuie un didactisme qui accompagne le spectateur dans la vision, comme quand le commentaire énonce "Les sacs sont fixés sur un poteau par un étrier » alors que deux plans montrent exactement à l'image cette opération.

Dans ce style de réalisation, le commentaire prévaut sur les images à tel point que l'on peut dire que celles-ci ne font qu'appuyer et confirmer ce qui est communiqué par la voix-off.

Une note différente est tout de même à signaler dans la voix-off de Night Mail lorsqu'elle interprète une comptine en rimes poétiques ${ }^{6}$, rythmées par une musique-off qui évoque les sonorités du train.

Terre d'Espagne (1937) de Joris Ivens est presque devenu un emblème du film documentaire politiquement engagé, ce à quoi contribue sensiblement le commentaire rédigé et récité par Ernest Hemingway.

25 "Ce commentaire de Hemingway a fait école, souligne Ivens. Il ne double pas l'image, reste en-deçà d'elle et lui donne plus de force en créant une relation dialectique entre le mot et l'image ${ }^{7}$ ».

26 En effet, il s'agit souvent d'informations sur la situation historique impossibles à déduire des seules images, mais dont celles-ci se nourrissent.

27 Il s'agit de témoigner du combat des Républicains pour la liberté et la justice contre les fascistes, durant la guerre civile espagnole - selon Ivens mieux identifiée comme « un putsch militaire contre un régime démocratique issu d'élections ${ }^{8} \%$.

Il est intéressant de savoir que la voix-off de Terre d'Espagne avait été, dans un premier temps, confiée à Orson Welles. Il est possible de comparer les deux versions bien que cette première n'ait été jamais distribuée car la diction de Welles semblait trop paisible, voire trop narrative, tirant le film du côté du fictionnel. Hemingway interprète donc lui-même son commentaire d'un ton qui le rend plus dramatiquement réaliste. En la quasi-absence des sons du réel, les images sont accompagnées par une musique-off (montage de plusieurs airs et chansons) qui occupe toute la scène sonore quand la voix-off - ou les tirs d'armes à feu, bombes, etc. - se taisent. À d'autres moments, la voix-off assume le rôle de traductrice des paroles diégétiques (orales ou écrites) mais d'une manière très imprécise, davantage en fonction de son propre récit, qui s'adresse avant tout au public américain pour lequel le film a été conçu. Lors des assemblées publiques, le commentaire couvre même la voix des intervenants, dont elle préfère donner les profils en tant que combattants et militants, avant d'en laisser entendre brièvement la pure sonorité. Et même quand des sous-titres sont utilisés, ceux-ci ont davantage la fonction de donner des informations contextuelles que celle de traduction. Il faut, qui plus est, signaler que dans la version française du film, la voix-off originale est doublée, ce dont - semblerait-il Ivens n'a pas été très satisfait.

29 Ce n'est sans doute pas un hasard si la même année (1937), le Républicain Luis Buñuel - qui après la défaite émigrera aux Etats-Unis - achève un film documentaire de dénonciation sociale, Las Hurdes-Terre sans pain. La voix-off récite avec un style très dur et objectivant un commentaire sur les conditions de vie d'une population extrêmement pauvre d'Espagne, ravagée par la misère et les maladies. 
Une apparente objectivité, parodiant celle des films zoologiques ou touristiques, couvrit son dur réquisitoire. Aujourd'hui, Terre sans pain explique et annonce la guerre civile durant laquelle les phalangistes fusillèrent l'ami de Buñuel, le poète Garcia $\operatorname{Lorca}^{9}$ (...). symphonie de Brahms, et la voix-off. Concernant l'oralité de celle-ci, il faut préciser qu'il existe plusieurs versions de ce film, même en français, avec des voix de narrateurs différents.

Il est, par ailleurs, intéressant de remarquer que le commentaire du film renforce le témoignage en intégrant l'expérience participative de l'équipe de tournage :

Dans une rue déserte, nous rencontrons une fillette, nous demandons ce qu'elle a et on nous répond que depuis trois jours l'enfant reste là sans bouger. Elle doit être malade parce qu'elle se plaint, mais nous ne parvenons pas à savoir ce qu'elle a. Un de nos amis s'improvise médecin, s'approche d'elle pour savoir ce qui lui fait mal à la gorge, il lui demande d'ouvrir la bouche, et nous pouvons voir ses gencives et sa gorge enflammées. Malheureusement, nous ne pouvons rien faire pour elle. Deux jours après, comme nous demandions de ses nouvelles, on nous a répondu que la fillette était morte. fait le récit.

Au-delà des spécificités artistiques et de contenu qui les distinguent, ces films documentaires de l'époque «classique » ont en commun le recours à un même style de mise en scène avec une dominance de sons extradiégétiques, voix-off et musique de fond.

On peut encore citer le remarquable commentaire poétique en voix-off du film Le chant $d u$ styrène (1958) d'Alain Resnais. Commandité par le groupe industriel Pechiney, ce film est un exemple de parfaite concordance stylistique entre images, musique, texte et mode d'énonciation de la voix-off. Sur une musique composée pour le film par Pierre Barbaud ${ }^{10}$, le commentaire écrit par Raymond Queneau en alexandrins ${ }^{11}$ est récité par Pierre Dux avec l'emphase qu'il lui faut :

Ô temps, suspends ton bol, ô matière plastique d'où viens-tu ? Qui es-tu ? Et qu'estce qui explique tes rares qualités ? De quoi es-tu donc fait? Quelle est ton origine? En partant d'un objet retrouvons ses aïeux! Qu'à l'envers se déroule son histoire exemplaire (etc.).

Appliqué à un contenu a priori improbable, la fabrication industrielle d'un bol en plastique, ce style transforme un film de commande d'entreprise en une œuvre d'art cinématographique à l'esthétique très recherchée. Ce qui n'a d'ailleurs pas aisément rencontré l'approbation du commanditaire...

\section{Le cinéma direct versus le commentaire en voix-off}

L'un des aspects marquants du nouveau cinéma documentaire de la fin des années Cinquante, appelé « cinéma direct », est la prise directe du son, ce qui entraîne, dans ce courant cinématographique, une distanciation nette vis-à-vis de l'utilisation de la voixoff, voire son abandon. De plus, tout élément extradiégétique à fonction de commentaire est également mis à l'écart, notamment la musique de fond. On peut y lire une réaction au documentaire « classique » qui reléguait les sons réels à une simple fonction d'ambiance. 
Ce sont des innovations techniques - l'apparition de la caméra légère portative $16 \mathrm{~mm}$, et ses successives améliorations, combinée avec celles du magnétophone, dont le fameux Nagra - qui déterminent non seulement un changement dans le style des prises de vue mais aussi, voire surtout, la possibilité d'enregistrer directement sur le lieu du tournage le son de manière synchrone avec les images.

Donner la parole aux gens filmés, réduisant ainsi le poids de l'auteur du film par le commentaire, devient l'une des stratégies de mise en scène du cinéma direct - de Michel Brault au Canada, de Jean Rouch en France, de Richard Leacock (qui fut l'assistant de Flaherty dans sa dernière réalisation) aux États-Unis et de bien d'autres réalisateurs.

Des prises de vues plus près de l'événement, pour l'image comme pour le son, et l'effacement, relatif, bien entendu, du cinéaste - qui est presque toujours lui-même cameraman dans ce style de réalisation-, sont autant de traits qui caractérisent l'approche du cinéma direct, permettant d'ailleurs beaucoup d'échanges entre cinéastes. Cette communauté stylistique n'enlève rien à la singularité de chacun, et il y a autant de déclinaisons de cinéma direct que de réalisateurs qui le pratiquent. Les plus radicaux, notamment aux États-Unis, prônent un cinéma où l'observation du réel et sa restitution (visuelle et sonore) priment sur toute autre motivation de l'auteur, lequel tend à se faire tellement discret que sa présence durant le tournage - selon l'expression que l'on attribue à Leacock - « n'est pas plus perçue que celle d'une mouche sur le mur ».

En France, le cinéma direct se décline avec l'œuvre de Jean Rouch en « cinéma-vérité ", dont l'emblème demeure son film Chronique d'un été (1961), co-réalisé avec Edgar Morin.

Au début du film, la voix-off de Rouch annonce : «ce film n'a pas été joué par les acteurs mais vécu par des hommes et des femmes... ».

42 C'est tout ce qu'il y a comme voix-off, une brève introduction au style "cinéma-vérité " du film, dans lequel, d'ailleurs, il n'y aura plus de commentaire : dans la véritable vie, il n'y a pas de commentaire.

43 Notons que ce que dit Rouch, tout de suite après, en prise directe et à l'image, est significatif, même pour ce qui est de la voix-off, car il souligne l'une des difficultés majeures que rencontre le cinéma du réel - ou de «non-fiction" comme certains préfèrent l'appeler - qui consiste à enregistrer les dialogues des personnes filmées. Cette difficulté peut être l'une des raisons du si fréquent recours à la voix-off dans le documentaire social.

Rouch dit : « ... je ne sais pas si l'on arrivera à enregistrer une conversation aussi normale qu'elle le serait s'il n'y avait pas de caméra ».

La difficulté tient à la "profilmie ${ }^{12}$ », raison exposée ici par Rouch, mais aussi aux entraves de l'enregistrement $\mathrm{du}$ son direct, qui demeurent malgré les avancées techniques du cinéma direct et celles de la successive vidéographie.

Le cinéma direct se développe donc, en principe, sans commentaire, voire contre le commentaire. Jean Rouch disait que le commentaire vieillit plus vite que les images, qui au contraire, acquièrent avec le temps une plus grande valeur documentaire. Il citait souvent à ce propos le film La Taranta (1962), réalisé par Gianfranco Mingozzi avec la collaboration scientifique de l'ethnologue Ernesto De Martino. Le film traite des danses de possession dans le sud de l'Italie (Pouilles), et les musiques proviennent des enregistrements effectués sur le terrain par l'ethnomusicologue Diego Carpitella. Le commentaire en voix-off est remarquablement écrit par le poète Salvatore Quasimodo. 
Une double stratégie caractérise, dans cette mise en scène, le traitement de la voix-off. Dans certaines séquences, elle est dominante sur des images reléguées au rôle d'illustration de son texte, tandis que durant les danses de possession, le commentaire en voix-off est beaucoup plus espacé, et devient donc plus discret dans sa co-occurrence avec les images et les sons du réel.

Au-delà de quelques problèmes de synchronisation dans le son direct de ce film, on éprouve certaines difficultés dans la compréhension des quelques paroles émises par les protagonistes. Ainsi, la voix-off en prend quelquefois en charge l'explication. Par exemple : «La possédée a demandé à saint Paul si elle doit poursuivre sa torture rythmée ou si la grâce du repos lui a été accordée ».

Concernant la critique de Rouch (qui par ailleurs appréciait beaucoup ce film) envers ce commentaire, on peut relever l'aspect daté de certaines interprétations ethnologiques, peut-être amplifié par l'admirable traduction littéraire : «La tarentulée se fait araignée. Elle devient l'araignée qui est en elle. Sa pensée devient rythme pur et de ses mouvements presque mécaniques surgissent des figures de libération, parcourues encore d'ombres désespérées ».

0 On a donc souvent entendu dire, par les cinéastes du cinéma direct - entre autres -, que le commentaire « tue les images", leur ôtant toute autonomie de signification. Comme nous le dit Claudine de France, dans la triade réalité/image/commentaire, ce dernier a la capacité d'influencer grandement la perception du spectateur: «(...) s'il a besoin de choses autres que lui pour exister, le commentaire peut avoir simultanément le "dernier mot" sur toute chose ${ }^{13}$ ».

Dans un passage bien connu de son film Lettre de Sibérie (1958), Chris Marker prouve qu'un commentaire peut faire dire ce qu'il veut aux images, reléguées au rôle de simple illustration d'un discours. La démonstration de Marker fonctionne un peu comme l'« effet Koulechov ${ }^{14}$ » appliqué à l'association «images-commentaire en voix-off » au lieu de « images-images».

52 Marker réalise trois montages en associant un commentaire à chaque fois différent à la même séquence d'images. La séquence montre des images d'une ville de Sibérie à l'époque soviétique: autobus, immeubles, voitures, ouvriers nivelant le sol de la rue à genou, un passant. Aux trois montages reconnus pour cette démonstration de Marker, on pourrait ajouter celui dans lequel la voix-off annonce cette expérience, car la séquence d'images est à peu près la même :

Je regardais autour de moi : un travail, une énergie et un enthousiasme indéniables. Un très lourd passé et une éclatante confiance en l'avenir. D'énormes lacunes et la ferme volonté de le combler. En enregistrant aussi objectivement que possible ces images de la capitale Iakoutsk, je me demandais franchement à qui elles feraient plaisir, puisqu'il est bien entendu que l'on ne saurait traiter de l'URSS qu'en termes d'enfer ou de paradis. Par exemple...

53 Et ici commence la démonstration avec le premier commentaire qui glorifie le régime socialiste :

Iakoutsk, capitale de la République socialiste soviétique de Iakoutie, est une ville moderne, où les confortables autobus mis à la disposition de la population croisent sans cesse les puissantes Zim, triomphe de l'automobile soviétique. Dans la joyeuse émulation du travail socialiste, les heureux ouvriers soviétiques, parmi lesquels nous voyons passer un pittoresque représentant des contrées boréales, s'appliquent à faire de la Iakoutie un pays où il fait bon vivre. 
« Ou bien » la deuxième voix-off qui exprime exactement le contraire :

Iakoutsk, à la sinistre réputation, est une ville sombre, où tandis que la population s'entasse péniblement dans des autobus rouge sang, les puissants du régime affichent insolemment le luxe de leurs Zim, d'ailleurs coûteuses et inconfortables. Dans la posture des esclaves, les malheureux ouvriers soviétiques, parmi lesquels nous voyons passer un inquiétant Asiate, s'appliquent à un travail bien symbolique : le nivellement par le bas.

Ou simplement: à Iakoutsk, où les maisons modernes gagnent petit à petit sur les vieux quartiers sombres, un autobus moins bondé que ceux de Paris aux heures d'affluence, croise une Zim, excellente voiture que sa rareté réserve aux services publics. Avec courage et ténacité, et dans des conditions très dures, les ouvriers soviétiques, parmi lesquels nous voyons passer un Iakoute affligé de strabisme, s'appliquent à embellir leur ville, qui en a bien besoin.

Et à l'auteur d'ajouter :

Mais l'objectivité non plus n'est pas juste. Elle ne déforme pas la réalité sibérienne, mais elle l'arrête, le temps d'un jugement...

Il faut encore souligner les autres sons co-occurrents avec la même séquence d'images et la voix-off: la musique extradiégétique, elle aussi à chaque fois différente s'accordant émotionnellement avec la signification du commentaire dans les premiers montages, et les sons du réel pour la dernière séquence où le style « objectif prime ».

\section{L'autocommentaire en voix-off}

57 Donnant la parole aux gens filmés, le cinéma direct développe en même temps l'autocommentaire ${ }^{15}$, abondamment utilisé depuis dans le cinéma documentaire et ethnographique. L'autocommentaire a un statut particulier dans l'analyse qui, dans le cinéma du réel, partage ce qui est off - extradiégétique - et ce qui est in - diégétique, dans le champ ou hors-champ. Ses formes de mise en scène sont multiples et il s'agit juste ici de rappeler qu'une voix-off peut être le fait d'un autocommentaire, spontané ou induit par le réalisateur-enquêteur.

La voix-off d'autocommentaire est une voix-in à certains moments du film, et le locuteur sera donc clairement identifiable pour le spectateur, car montré à l'image.

Mais comment analyser le cas de la situation d'entretien, très répandu - quelle qu'en soit la forme - dans le cinéma documentaire ? Souvent le réalisateur - qui peut être lui-même l'enquêteur, voire le cameraman - n'est jamais montré à l'image bien que l'on puisse entendre sa voix. L'espace diégétique de l'entretien suppose la présence de la caméra et le spectateur la ressent. Cela ne tient pas qu'au fait que, dans le documentaire, on ne respecte plus l'interdiction du « regard caméra ». C'est surtout cette parole dirigée vers l'autre (l'enquêteur ou le spectateur), générée ou relayée par le tournage qui fait reconnaître l'autocommentaire, qu'il soit présenté en situation d'entretien ou non.

C'est une parole profilmique, qui ne surgirait pas sans la présence de la caméra, qui est en conséquence ressentie comme présente. Quand ces voix impliquées dans l'autocommentaire deviennent off - et non seulement hors-champ-, il y a coprésence (dans l'esprit du spectateur) de deux scènes filmiques diégétiques qui se développent parallèlement, dont l'une assume une fonction de commentaire et de narration vis-à-vis de l'autre que l'on montre en images.

Cahiers de Narratologie, 20 | 2011 
61 La cinématographie scientifique, mais aussi de vulgarisation, utilise beaucoup, par exemple, une mise en scène qui donne la parole à l'expert du sujet traité en le montrant de temps en temps à l'image. C'est une parole qui a un statut d'autorité, comme celle de tout commentaire en voix-off, mais dont le texte est oral et l'auteur montré à l'image.

Dans l'autocommentaire direct, la personne émet pendant le tournage un commentaire sur les activités montrées - souvent les siennes, tout en les faisant - et sa voix peut, de temps en temps, devenir non seulement hors-champ, mais véritablement off. Dans d'autres cas encore, on peut reconnaître dans la voix-off celle d'une personne filmée énonçant un autocommentaire sur les images montrées mais ce commentaire a été enregistré a posteriori, souvent en regardant les images du film - situation qui n'est pas forcément montrée à l'image.

63 Même en ce qui concerne la voix-off, les formes d'autocommentaire sont, comme on l'a dit, très variées dans le cinéma documentaire.

64 Le cas de la voix intérieure est particulier, car tout en n'étant pas audible en situation réelle, elle appartient au personnage diégétique montré dans le film.

65 Cette mise en scène est extrêmement rare en cinéma documentaire, car on s'approche d'un dispositif très fictionnel. Cependant, on peut en signaler un exemple dans un film ethnographique de Jean-Pierre Olivier de Sardan: La vieille et la pluie (1974). Il s'agit vraisemblablement d'une voix enregistrée lors d'un entretien (que l'on voit au début du film) mais elle est montée en off sur les images de la vieille femme qui parcourt seule un long trajet pour aller au marché Et naturellement, l'image montre qu'elle ne parle pas même si on entend sa voix, qui peut ainsi être interprétée comme sa voix intérieure.

Enfin, une voix-off subjective, à la première personne mais jamais à l'image, peut être considérée comme une forme d'autocommentaire dans le cas où le locuteur participe de manière évidente à la diégèse - c'est-à-dire que sa présence au moment du tournage (par la voix ou par des indices visibles) est attestée pour le spectateur, bien que son image soit toujours cachée, derrière la caméra, par exemple, dans le cas du cinéaste lui-même.

\section{Le commentaire en voix-off dans un film ethnographique de Jean Rouch}

Nonobstant l'attitude critique du cinéma direct vis-à-vis du commentaire, Jean Rouch inscrit sa voix-off dans la grande majorité de ses films et il n'est pas le seul à le faire.

Prenons en exemple un film parmi les plus ethnographiques de Rouch, Le Dama d'Ambara (1974), qui présente un texte en voix-off aux multiples facettes, dont certaines assez étonnantes et par là même intéressantes.

Chez les Dogons du Mali, le Dama est un rituel funéraire de levée de deuil. Il fait partie des cérémonies de masques étudiées sur le terrain dans les années Trente par l'ethnologue Marcel Griaule ${ }^{16}$ pour en faire ensuite sa célèbre thèse d'ethnologie, Masques Dogons, soutenue en 1938. Griaule avait également réalisé un film à ce sujet : Sous les masques noirs (1938), où le style « colonial » du prépondérant commentaire en voix-off tient plus au ton de l'énonciation orale qu'au texte lui-même. Renforce ce style une musique de fond génériquement exotique qui n'a rien à voir avec les musiques exécutées par les Dogons lors des danses rituelles, et ce malgré la tentative de synchronisation destinée à suggérer un son direct. Rien à voir non plus avec d'autres musiques qui pourraient faire partie de 
la même aire culturelle. Musique et commentaire off correspondent non pas à une représentation du réel mais à la représentation de ce que l'on supposait être, et l'on cultivait à être, le goût du public de l'époque vis-à-vis de ce réel africain. Était-ce vraiment ce que Griaule désirait communiquer avec ce film, alors que l'on connaît la qualité de son travail d'écriture ethnologique? On a le droit d'en douter. Et en effet, Philippe Lourdou se demande : «Qui a rédigé le commentaire ? (...) Le "parleur" (...) a-t-il été choisi par le réalisateur du film ou imposé par les producteurs ${ }^{17}$ ?».

Or, bien que Griaule puisse être considéré comme un précurseur dans l'utilisation de la caméra comme outil de documentation scientifique, et surtout ait incité d'autres, notamment Rouch ${ }^{18}$, à le faire, la mise en scène, surtout sonore, de ses deux films sur les Dogons nous autorise à penser qu'il ne se souciait pas trop de la communication et de la mise en scène filmiques. Marcel Griaule ne maîtrisait probablement pas les étapes de la réalisation qu'il confiait donc entièrement aux professionnels du cinéma : il ne filme pas lui-même et il n'a vraisemblablement pas participé au montage. En conséquence, il faut le considérer plus comme un consultant scientifique que comme le réalisateur du film, bien qu'il soit ainsi crédité aux génériques.

71 Extraordinaires par leur valeur documentaire sont, en revanche, les images de Sous les masques noirs de Griaule, qui apportent un complément important à ses descriptions ethnographiques écrites, bien que la cérémonie funéraire filmée soit probablement une reconstitution.

Presque 40 ans après, Jean Rouch filme la même cérémonie funéraire (Dama) faite en l'honneur de Ambara Dolo qui avait été l'un des principaux informateurs de Griaule, et réalise ce film (Le Dama d'Ambara) avec Germaine Dieterlen, continuatrice des recherches de Griaule chez les Dogons. Le Dama dure trois jours, pendant lesquels les nouveaux masques peints et ornés défilent et dansent pour enchanter les âmes des morts et les acheminer vers leur nouvelle demeure.

Pour ce film, les auteurs élaborent un commentaire en grande partie tiré du livre de Griaule. Jean Rouch en parle ainsi : « ...cela m'a permis de mettre sur ces images (...) un texte admirable de Griaule sur l'analyse de ce que représente le Dama ${ }^{19}(. .)$.$» .$

Comme les autres films nés sous le signe de l'héritage ethnographique de Marcel Griaule et réalisés chez les Dogons avec Germaine Dieterlen, Le Dama d'Ambara est l'un des plus réfléchis à l'avance ${ }^{20}$, des moins improvisés, de la multiforme cinématographie de Rouch. De même, ce n'est pas dans ses habitudes qu'un texte écrit précède l'image et la parole du terrain.

Le film résulte donc d'un ensemble de compétences ethnographiques réunies dans un «texte » audio-visuel complexe, dont la narration est assurée par le commentaire en voix-off centré sur des textes choisis de Griaule. Mais nous allons voir que plusieurs fonctions sont prises en compte par cette voix-off.

Dès les premières images, Rouch s'identifie à travers la voix-off comme l'auteur du film énonçant : « ... ainsi fut tourné ce film Le Dama d'Ambara réalisé avec Germaine Dieterlen sur des textes de Marcel Griaule ». Par ce "réalisé avec », la voix off est ainsi d'emblée présentée au spectateur comme celle du (co-)réalisateur. Or, la voix de Rouch est, pour un public averti, une marque très reconnaissable qui personnalise la plupart de ses films. Cela renforce une sorte de complicité entre lui-même en tant qu'auteur et son public - celui qui connaît au moins une partie de sa cinématographie. Bien sûr, on peut aussi reconnaitre assez vite un film de Rouch par le style des prises de vue, mais sa voix en off 
accélère et facilite cette reconnaissance : c'est à chaque nouveau film une retrouvaille. Or, cette utilisation de la voix-off comme marque d'auteur ne relève pas, comme on pourrait le croire, d'un excès de vanité, mais plutôt d'une assomption de responsabilité, et bien d'autres auteurs de la cinématographie ethnographique optent pour cette mise en scène personnalisée de la voix-off.

La voix-off de Rouch assume ici, et d'une manière générale dans ses commentaires, un ton de très suggestif, séduisant, presque rêveur, qui peut l'assimiler à celle du narrateur d'un conte. Cependant, le spectateur est également captivé par le réalisme du tournage et il est très important de souligner que chez Rouch, le choix de mise en scène avec une voix-off importante n'exclut pas les sons directs, parmi lesquels émergent dans ce rituel du Dama les musiques tambourinées et les cris des danseurs masqués.

Tout de suite après l'introduction des auteurs du film, Rouch, en voix-off, prend en charge une autre fonction, celle du doublage de la voix-in du fils d'Ambara Dolo, apparu entre-temps à l'image. Il devient donc traducteur au sens strict du terme, même si ces traductions du son direct sont souvent plus une élaboration en fonction du récit filmique qu'une restitution fidèlement littérale.

79 Arrivent ensuite les textes tirés de l'ouvrage de Griaule dont le choix est multiforme et par là même particulièrement intéressant.

80 Dans un premier temps, la voix-off énonce le mythe fondateur du Dama où le commencement de la mort pour le genre humain est associé au don divin de la parole. Est évoquée pour la première fois la figure emblématique de la mythologie des Dogons rapportée par Griaule et ses continuateurs: le renard pâle, maître du désordre et inventeur du premier masque.

81 Ce texte est déroutant pour tout public non-initié, car il introduit d'emblée, sans plus d'explications, un univers symbolique dont le spectateur est loin d'avoir les codes. La voix-off prend ensuite en charge une fonction descriptive du rituel funéraire chez les Dogons, ce qui devrait être davantage compréhensible ; toutefois, il n'en est rien pour les spectateurs car la description des activités est imbriquée dans les croyances qui les génèrent.

83 Quand le défilé des masques commence, le commentaire les identifie brièvement avec leurs attributs symboliques, mais tout de suite après introduit un autre niveau de contenu textuel qui évoque un dialogue sur le terrain entre Griaule et son informateur. La voix-off de Rouch se charge donc d'interpréter la voix de Griaule (" et Griaule dit : etc. »), lequel en évoquant sa forte impression face aux masques du Dama en décrit quelques détails. Peut-être pour ne pas anticiper ce que le spectateur verra un peu plus tard dans le film, Rouch n'a pas choisi de doubler à l'image ces détails donnés par le commentaire, qu'il laisse donc se dérouler sur des plans d'ensemble. Ce sera par la suite que la voix-off se chargera de canaliser l'attention du spectateur vers quelques détails des masques dansants en décryptant leur portée symbolique et rituelle.

Et puis, on arrive à ce moment magique et étonnant où la voix-off déclame, traduites en français, les devises mythiques des masques (que Griaule avait transcrites suivant ses informateurs). Au début de cette séquence la voix-off semble traduire une déclamation synchronique avec les événements montrés mais elle prend ensuite son autonomie, tout en gardant un lien de signification avec les images. Ces récits mytho-poétiques sont très suggestifs bien que la grande majorité du public censé voir ce film ne puisse guère comprendre leur réelle signification. Ce choix de textes pour la voix-off est très 
intéressant car il confie à la voix le même niveau de communication esthétique mystérieuse - car hermétique pour les non-initiés - que porte la vision à l'image des danses de masques. Bref, on n'y comprend probablement rien, mais on est fasciné.

\section{BIBLIOGRAPHIE}

\section{Bibliographie}

COMOLLI, Annie, 1995, « La parole retrouvée ou l'autocommentaire et ses mises en scène », Xoana . Images et sciences sociales, $\mathrm{n}^{\circ} 3$. (pp. 99-113).

CHION, Michel, 2005, La voix au cinéma, Cahiers du cinéma (1982), Editions de l'Etoile, Paris.

Découvrir les films de Jean Rouch, 2010, édition Archives françaises du film du CNC.

FRANCE, Claudine de, 1982, Cinéma et Anthropologie, Fondation de la Maison des Sciences de l'Homme, Paris, (deuxième édition, revue et augmentée, 1989).

FRANCE, Claudine de, 1995, « Le commentaire, rival de l'image dans la mise en scène du réel » Xoana Images et sciences sociales $\mathrm{n}^{\circ}$ 3. (pp. 73-88).

GUERRONNET, Jane, 1995, « Le commentaire des films muets : du hors scène à la scène ", Xoana Images et sciences sociales $\mathrm{n}^{\circ}$ 3. (pp. 91-98).

LOURDOU, Philippe, 1994, «Le commentaire dans les films ethnographiques de Marcel Griaule » in Du film ethnographique à l'anthropologie filmique, (France C. de, Ed), Paris éditions des archives contemporaines. (pp. 127-157).

METZ, Christian, 1966, « La grande syntagmatique du film narratif » Communication $n^{\circ} 8$ (pp. 120-124).

RICHARD, Serge, 1976, « 3 histoires, 2 hommes, 1 film », L'unité, 9 janvier.

SADOUL, George, 1961, Histoire du cinéma, Flammarion, Paris.

SOURIAU, Etienne, 1953, L'Univers filmique, Flammarion, Paris.

SOURIAU, Etienne (Anne SOURIAU éd.), 1990, Vocabulaire d'esthétique, PUF, Paris.

\section{ANNEXES}

\section{Films cités (par ordre chronologique)}

Nanook of the North (1922) de Robert Flaherty

Le chanteur de Jazz (1927) d'Alan Crosland

L'Homme à la caméra (1929) de Dziga Vertov 
Night Mail (1936) de Harry Watt et Basil Wright

Las Hurdes (1937) de Luis Buñuel

Terre d'Espagne (1937) de Joris Ivens

Sous les masques noirs (1938) de Marcel Griaule

Cimetières dans la falaise (1952) de Jean Rouch

Le chant du styrène (1958) d'Alain Resnais

Lettre de Sibérie (1958) de Chris Marker

Chronique d'un été (1961) de Jean Rouch et Edgar Morin

La Taranta (1962) de Gianfranco Mingozzi

La vieille et la pluie (1974) de Jean-Pierre Olivier de Sardan

Le Dama d'Ambara (1974) de Jean Rouch et Germaine Dieterlen.

\section{NOTES}

1. «S'il est vrai que le montage conçu comme manipulation irresponsable, magique et toutepuissante est dépassé, le montage comme construction d'une intelligibilité au moyen de "rapprochements" divers n'est en aucune façon "dépassé", puisque le film est de toute façon discours (c'est-à-dire lieu de co-occurrence de divers éléments actualisés) ». Metz, C., « La grande syntagmatique du film narratif », p. 123.

2. Etienne Souriau en donne la définition pour la fiction cinématographique: «Tout ce qui est censé se passer, selon la fiction que présente le film ; tout ce que cette fiction impliquerait si on la supposait vraie ». Vocabulaire d'esthétique, p. 240 ; et encore : «Diégèse : tout ce qui appartient, “dans l'intelligibilité" (comme dit M. Cohen-Seat) à l'histoire racontée, au monde supposé ou proposé par la fiction du film ». L'Univers filmique, p. 7.

3. "A partir de quel seuil peut-on dire que la voix de quelqu'un est, si peu que ce soit, horschamp ou off ?". Chion, M., La voix au cinéma, p. 119.

4. En 1927, Le chanteur de Jazz d'Alan Crosland marque le début du «sonore », ou plutôt du " parlant».

5. Cf. Guéronnet, J., " Le commentaire des films muets : du hors scène à la scène ».

6. Écrite pour le film par le poète W.H. Auden: "This is the Night Mail crossing the border; Bringing the cheque and the postal order ; Letters for the rich, letters for the poor ; The shop at the corner and the girl next door (etc.)".

7. Richard, S., « 3 histoires, 2 hommes, 1 film ».

8. ibid

9. Sadoul, G., Histoire du cinéma, p. 204.

10. Connu comme le premier en France à utiliser systématiquement l'ordinateur pour la composition musicale, il est l'un des fondateurs du Groupe de Musique Algorithmique de Paris (GMAP) en 1959.

11. Vers de douze syllabes.

12. En anthropologie filmique, on entend par profilmie le changement de comportement des personnes filmées que l'on peut attribuer à la présence de la caméra. Cf. France, C. de, Cinéma et Anthropologie, p. 373.

13. France, C. de, «Le commentaire, rival de l'image dans la mise en scène du réel », p. 82. 
14. L'« effet Koulechov "- du nom du metteur en scène soviétique qui réalisa cette fameuse expérience en 1922, - consiste à montrer la même image en gros plan de l'acteur Mosjoukine - volontairement inexpressif - en la juxtaposant successivement à des images à chaque fois différentes. Les spectateurs soumis à cette expérience eurent l'illusion que l'acteur exprimait avec beaucoup de talent des sentiments aussi divers que la faim, la tristesse ou le désir. L'« effet Koulechov " prouve ainsi le rôle créateur du montage visuel.

15. Cf. Comolli, A., " La parole retrouvée ou l'autocommentaire et ses mises en scène ».

16. C'est sous la direction de Griaule que Jean Rouch achève en 1952 sa thèse d'État sur La religion et la magie Songhai.

17. Lourdou, P., « Le commentaire dans les films ethnographiques de Marcel Griaule », p. 128.

18. C'est Griaule qu'au début des années 1950 incite Jean Rouch à la réalisation de son premier film en pays dogon : Cimetières dans la falaise.

19. (propos recueillis le 8 juin 1995) Découvrir les films de Jean Rouch, p. 108.

20. Rouch et Dieterlen attendaient depuis longtemps l'occasion de filmer les rituels des masques décrits par Griaule, qui concernent également les films tournés entre 1967 et 1973 sur le cycle septénaire de cérémonies du Sigui, qui ont lieu tous les soixante ans.

\section{RÉSUMÉS}

Une analyse de la voix-off dans le cinéma documentaire - dont la fonction est avant tout le commentaire - où la relation texte/image est considérée comme fondamentale dans la genèse de la signification. Avec l'avènement $d u$ sonore, le commentaire occupe dans le cinéma documentaire «classique » une place prédominante, jusqu'à la fin des années Cinquante lorsque commence à s'affirmer la nouvelle stratégie de mise en scène du cinéma direct. Foncièrement critique envers le commentaire qu'il considérait comme trop chargé d'autorité, le cinéma direct instaure alors une autre relation avec le réel, restituant la parole aux protagonistes des actions représentées. L'analyse de la voix-off et du commentaire s'appuie sur des exemples cinématographiques - dont Flaherty, Buñuel, Ivens, Resnais, Marker, Rouch. De ce dernier, on analyse ensuite le commentaire mytho-poétique du film ethnographique Le Dama d'Ambara, considéré comme un exemple de la délicate quête d'équilibre entre l'expression artistique de la cinématographie et la communication scientifique de l'anthropologie.

Un'analisi della voce-off nel cinema documentario, dove assicura innanzitutto una funzione di commento, prendendo come fondamentale nella produzione di significato la relazione testo/ immagine. Con l'avvento del cinema sonoro, il commento assume nel documentario « classico » un'importanza predominante, fino alla fine degli anni cinquanta, quando comincia ad affermarsi la nuova strategia di realizzazione del cinema diretto. Fondamentalmente critico verso il commento che considera troppo carico d'autorità, ma anche della musica di sottofondo, il cinema diretto instaura una diversa relazione con il reale, restituendo la parola ai protagonisti dell'azione rappresentata. L'analisi della voce-off e del commento si appoggia su alcuni esempi cinematografici - tra cui Flaherty, Buñuel, Ivens, Resnais, Marker, Rouch. Di quest'ultimo si analizza poi il commento etno-poetico del film Le Dama d'Ambara, come esempio della delicata ricerca d'equilibrio tra l'espressione artistica della cinematografia e la comunicazione scientifica dell'antropologia. 
INDEX

Mots-clés : analyse, anthropologie, autorité, cinéma, commentaire cinématographique, diégétique, documentaire, film ethnographique, montage, musique, mythe, oralité, récit, représentation, rituel, Rouch, voix-off

\section{AUTEUR}

SILVIA PAGGI

Professeur d'anthropologie filmique à l'Université de Nice-Sophia Antipolis, Laboratoire CIRCPLES 